\title{
Radiographic damage in hands and wrists of patients with juvenile idiopathic arthritis after 29 years of disease duration
}

\author{
Anne M. Selvaag ${ }^{1 *} \mathbb{D}$, Eva Kirkhus ${ }^{2,3}$, Lena Törnqvist ${ }^{2,4}$, Vibke Lilleby ${ }^{1}$, Hanne A. Aulie ${ }^{1,5}$ and Berit Flatø $\varnothing^{1,3}$
}

\begin{abstract}
Background: There are few studies on radiographic outcome after long-term disease duration in juvenile idiopathic arthritis (JIA). We wanted to evaluate 29-year radiographic outcome in hands/wrists and predictors of damage in patients with long-term active JIA.

Methods: Patients diagnosed from 1980 to 1985, who had active disease at 15-, 23- or 29-year follow-up and arthritis in the wrists during the disease course, were reexamined with radiographs of hands/wrists. We used the adapted version of the Sharp van der Heijde (aSvdH) score and Carpal Height Ratio (CHR) to evaluate radiographic outcome.

Results: Sixty patients, mean age 38 years, were reexamined at median 29-year follow-up. 33 patients (55\%) had an aSvdH score $>0$, median score was 4.0 (range $0-313$ ), and $25 \%$ of the scores were high ( $\geq 53$ ). Most patients with radiographic damage (88\%) had both erosions and JSN. 52\% of the patients had damage in the wrists, $43 \%$ in the MCP joints and 40\% in the PIP joints.

The CHR correlated strongly with the aSvdH. Both scores had high correlations with the Juvenile Arthritis Damage Index and the number of joints with limited range of motion (LROM) $\left(r_{s}=-0.688\right.$ to $\left.0.743, p \leq 0.001\right)$. The aSvdH correlated weakly with measures of disease activity.

The number of joints with LROM, ESR and the HAQ disability score at 15 years and HLAB27 positivity predicted the aSvdH score and the CHR at 29-year follow-up.

Conclusions: The majority of patients with long-term active JIA had modest radiographic damage, but more frequently in wrists than in fingers. The radiographic scores correlated well with measures of disease damage. Restricted mobility in joints at 15 years was the most important predictor of radiographic damage at 29 years.
\end{abstract}

Keywords: Radiography, Juvenile idiopathic arthritis, Follow-up studies, Disease progression, Prognosis, Risk factors, Longitudinal studies

\section{Background}

Juvenile idiopathic arthritis (JIA) is a chronic inflammatory autoimmune disease which affects the joints of a child. This chronic inflammation can cause growth disturbances, joint space narrowing (JSN) and erosions in the joints. Patients with a polyarticular disease course have the highest probability of attaining joint destructions [1].

Until recently a standard for radiographic assessment in JIA did not exist. Several methods have been suggested

\footnotetext{
*Correspondence: anselv@ous-hf.no

'Department of Rheumatology, Oslo University Hospital, Rikshospitalet,

Post-box 4950 Nydalen, 0424 Oslo, Norway

Full list of author information is available at the end of the article
}

and evaluated [2-6]. One has looked for an index joint which could represent the radiographic status of a child with JIA, and found that the joints most often affected in polyarticular disease are the hands and wrists $[1,7-9]$.

Recently an adapted version of the Sharp/van der Heijde (aSvdH) score has been developed for radiographic scoring of patients with JIA [10]. It has proved to be reliable, has shown good construct validity and is capable of detecting radiographic progression [10]. In most late studies on radiographic outcome in patients with adult rheumatoid arthritis (RA), the method of Sharp and van der Heijde is frequently applied with a 
focus on hands and feet. We therefore chose to use the $\mathrm{aSvdH}$ method to score radiographs of hands/wrists in our adult JIA group.

The Poznanski score, an expression of damage in the carpal area, has been used in several studies of radiological damage of hands/wrists in JIA $[5,6,11,12]$. The Poznanski score is dependent on a growth plate in the distal radius and thus not suitable for adults. However, the Carpal Height Ratio (CHR) has some similarities with the Poznanski score, assesses carpal damage and is applicable in adults $[13,14]$.

Nutritional factors which influence mineral homeostasis, differentiation of the osteoblasts and the immune system, such as vitamin D and vitamin $\mathrm{A}$, have not previously been included in long-term studies of radiographic outcome in JIA $[15,16]$.

There is a lack of long-term radiographic outcome studies of adult patients with JIA. The aim of our study was to describe radiographic damage in hands/wrists of patients with long-term active JIA after 29 years of disease duration, by applying the aSvdH score and the $\mathrm{CHR}$ in these patients. We also wanted to assess correlations between radiographic outcome and disease variables after 29 years and evaluate possible predictors of radiographic outcome.

\section{Methods \\ Patients}

A total of 260 patients with juvenile idiopathic arthritis (JIA), first time referred to Oslo University Hospital, Rikshospitalet from 1980 to 1985, were reexamined clinically after median 15 years of disease duration and by mailed questionnaires after median 23 years [17, 18]. A total of 176 of these patients were assessed with questionnaires after median 29.6 years [19]. Those who had signs of active disease either at 15,23 or 29.6 years came to an additional clinical examination. Long-term active disease was defined as having active disease at 15-year follow-up or later. Of the 90 patients examined with long-term active disease, 60 patients $(67 \%)$ had a history of previous or present arthritis in the wrists and underwent radiographs of hands/wrists.

The patients were classified according to the International League of Associations for Rheumatology (ILAR) criteria for JIA after 15 years of disease duration [18, 20]. Data from baseline was available to fulfil the criteria. Disease onset was defined as the day the physician diagnosed the arthritis.

The study was approved by the Regional Committee for Medical and Health Research Ethics. Written informed consent was obtained from all the participants according to the Declaration of Helsinki.

\section{Clinical examination}

The patients were examined by one of three pediatric rheumatologists at follow-up (BF, VL, AMS). The clinical examination included registration of number of joints with swelling, tenderness and limited range of motion (LROM), number of active joints (swelling or both tenderness and LROM), and physician's global assessment of disease activity [on a $10 \mathrm{~cm}$ Visual Analogue Scale (VAS), where 0 means no disease activity and 10 means very severe disease activity].

We used the Juvenile Arthritis Damage Index (JADI) to assess clinical damage, the Health Assessment Questionnaire (HAQ) and the Juvenile Arthritis Disease Activity Score (JADAS-71) to evaluate physical disability and disease activity and the Medical Outcome Study 36item Short Form Health Survey (SF-36) to assess quality of life [21-24]. The preliminary criteria for remission in select categories of JIA was applied as described in a previous study on the same cohort [19].

Blood samples were drawn after an overnight fast. Erythrocyte sedimentation rate (ESR), C-reactive protein (CRP), rheumatoid factor (RF) IgM, anti-cyclic citrullinated peptides (anti-CCP) and antinuclear antibody (ANA) were analysed by standard methods. Vitamin A was measured by ultra high-performance liquid chromatography with UV detection and 25-hydroxy vitamin D was measured by liquid chromatography-tandem mass spectrometry.

\section{Radiographic examinations}

Conventional radiographs of the hand/wrist in the posteroanterior view, digital technique, were assessed in consensus by two musculoskeletal radiologists experienced in radiology of children with rheumatic diseases (EK, LT). The aSvdH method was used to score the radiographs of the hands/wrists [10]. The score is based on the assessment of joint space narrowing in 15 areas and bone erosions or deformity in 21 areas, on a $0-4$ and a $0-5$ point severity scale, respectively. Bone erosions and deformity were considered as equivalent. The total aSvdH score is calculated as the sum of the scores for JSN (range 0-120) and erosion (range 0-210) and ranges from 0 to 330 . We defined a score above the 75 -percentile as a high score/severe damage.

The CHR was calculated as the ratio between the carpal height (measured between the base of metacarpal III and the distal radial articular surface, measured along the axis of metacarpal III) and the length of metacarpal III [13]. The average CHR in normal controls (Belgian volunteers) has been found to be $0.52 \pm 0.07$ [14].

Six patients had signs of previous operations (prosthesis and/or arthrodesis) in joints scored by the aSvdH method 
and these joints were given the highest erosion and JSN sores.

Thus we obtained complete aSvdH scores for all the patients. CHR could not be scored in 7 patients due to joint fusion or prosthesis in the third carpometacarpal joint or in the third metacarpophalangeal (MCP) joint.

\section{Statistical analyses}

The differences between 2 patient groups were analyzed by $\mathrm{t}$ tests for continuous normality distributed data, Mann-Whitney $U$ test for non-normality distributed data and $\chi^{2}$ for frequencies. Because of non-normality distribution, we used the Kruskal-Wallis test to analyze differences in the aSvdH scores across the JIA categories. We used a one way analysis of variance with Bonferroni correction to look for differences in CHR between JIA categories. Spearman's correlation coefficient $\left(r_{s}\right)$ was used for non-normality distributed variables.

Missing data was observed in up to $7 \%$ of all variables except for JADAS-71 (10\%) and CHR scores (12\%). Missing data was replaced by median substitution except for CHR scores which were replaced by the worst recorded CHR as missing scores were due to bone damage in the wrists which made it impossible to score.

Initial univariate linear regression analyses tested the relation between patient characteristics. HLA alleles, disease variables at baseline, core set variables at 15-year follow-up and aSvdH or CHR scores at 29 years [25]. Subsequent multivariate linear regression analyses were used to identify a set of statistically significant predictors, where variables with a $\mathrm{p}$ value $<0.05$ in the univariate tests were analyzed as possible predictors. Highly intercorrelated independent variables $(r>0.7)$ were avoided. Backward and forward regression models were used.

For all analyses, $\mathrm{p}$ values $<0.05$ ( 2 tailed tests) were considered statistically significant. All analyses were performed on the SPSS software program (SPSS Inc., Chicago, IL, USA) version 21.0.

\section{Results}

\section{Patients}

Out of the original cohort of 260 patients, 60 patients with long-term active JIA and a history of previous or present arthritis in the wrists underwent radiographs of the hands and wrist joints after median 29.4 years (range 26-38) of disease duration (Fig. 1). We missed one patient, who had swelling in the wrist and two proximal interphalangeal (PIP) joints at 29 years, but underwent no radiographs of the hands, thus not included in the study. The 60 patients had significantly more affected wrists both at 6 months and 15-year follow-up compared to those 200 not examined $(p \leq 0.001)$. Physician's global, number of active joints, number of joints with LROM, HAQ, SF-36 PCS and patient's global were all significantly

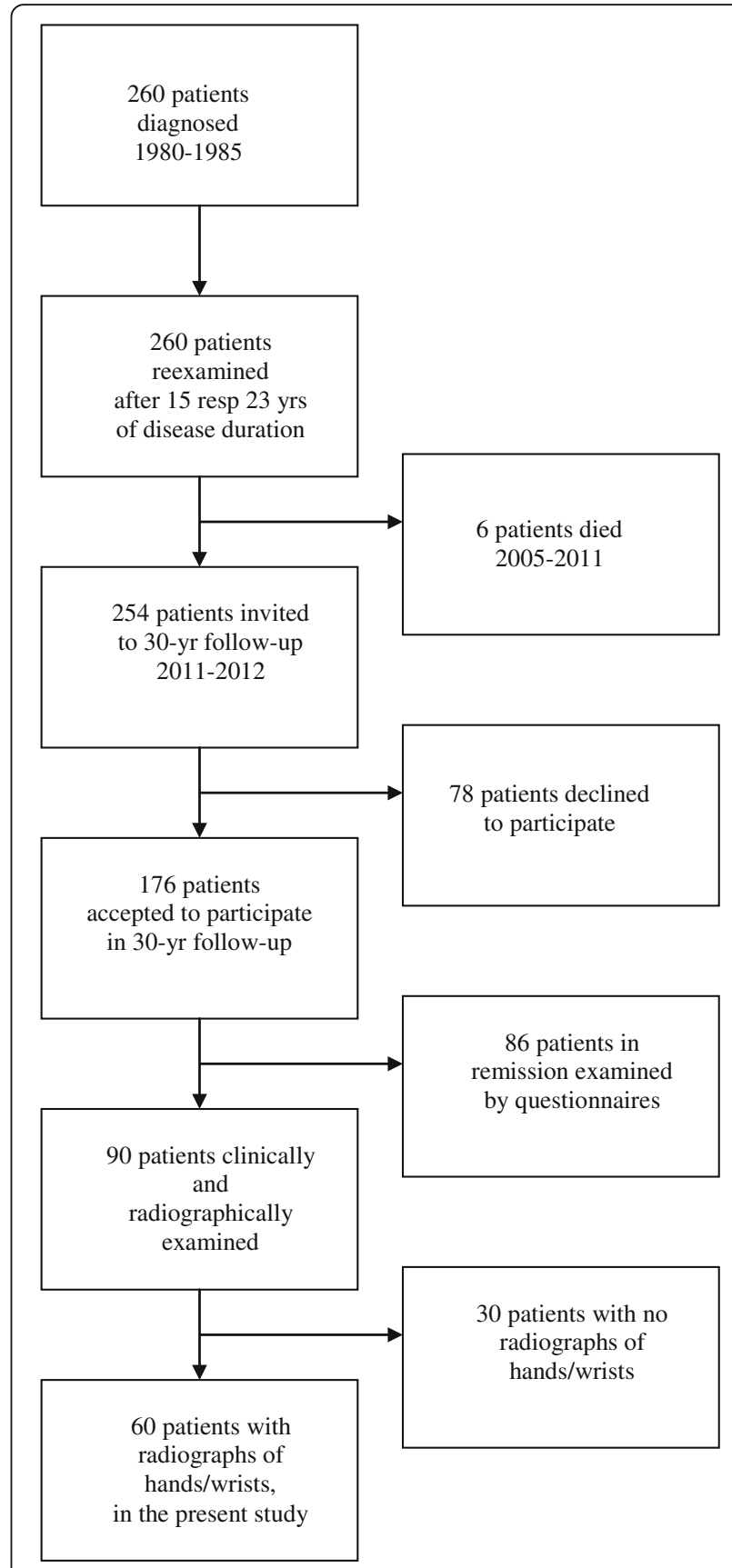

Fig. 1 Flow chart of how the 60 patients were selected from the original cohort of 260 patients

worse at 15-year follow-up in the study group than in the 200 patients not examined ( $p \leq 0.05$, data not shown).

Table 1 shows a description of the patient group. The patients had a median age of 38.2 years (range 30-45) and $70 \%$ were female. In general patients had modest disease activity (median 0 swollen joints, 4 joints with LROM, 0 active joints), but 48 patients (80\%) had active disease and only 12 patients (20\%) were in remission off medication at 29-year follow-up. Four patients (7\%) were 
Table 1 Demographic and clinical characteristics of 60 patients with JIA after 29 years of disease duration

\begin{tabular}{ll}
\hline Characteristics & Value \\
\hline Age, years & $38.2(30-45)$ \\
Female & $42(70)$ \\
Disease duration, years & $29.4(26-38)$ \\
Number of swollen joints & $0(0-12)$ \\
Number of tender joints & $1(0-16)$ \\
Number of joints with LROM & $4(0-56)$ \\
Number of active joints & $0(0-13)$ \\
PGA, VAS cm & $1.5(1-7.6)$ \\
Patient's global well-being, VAS cm & $2.0(1-5.9)$ \\
HAQ & $0.5(0-1.6)$ \\
JADAS & $4.8(0.2-27.1)$ \\
JADI total & $6(0-73)$ \\
CRP, mg/l & $2.0(0-38)$ \\
ESR, mm/h & $6.0(2-60)$ \\
Vitamin A umol/L & $1.6(0.9-2.4)$ \\
Vitamin D nmol/L & $64(26-100)$ \\
Patients in remission off medication & $12(20)$ \\
Patients with inactive disease on medication & $8(13)$ \\
Patients with persistently active disease & $40(67)$ \\
Patients on sDMARDs & $19(32)$ \\
Patients on biological immunosuppressants & $21(35)$ \\
Patients on glucocorticosteroids & $6(10)$ \\
\hline Employed & \\
Nam & $46(77)$ \\
\hline
\end{tabular}

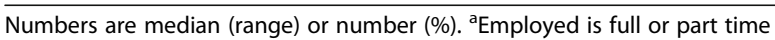
LROM limited range of motion, PGA physician's global assessment of disease activity, JADAS juvenile arthritis disease activity score, JADI juvenile arthritis damage index, sDMARDs synthetic disease-modifying anti-rheumatic drugs

RF IgM-positive, 6 patients (10\%) were anti-CCP-positive and 19 (32\%) were ANA positive.

\section{Radiographic scores of the hand and wrist after $\mathbf{2 9}$ years} Thirty three patients (55\%) had radiographic damage (aSvdH > 0), 9 patients (15\%) had aSvdH scores between 1 and 20, 9 patients (15\%) had aSvdH scores between 21 and 52, and 15 (25\%) had aSvdH scores $\geq 53$ (range 53-313).

The aSvdH scores of the radiographs of hands/wrists were median 4 (range $0-313$ ), and the scores of erosions and JSN were 1 (range 0-205) and 3 (range 0-110), respectively (Table 2). Patients with systemic or polyarticular RF-positive JIA had the highest frequency of damage. Patients with polyarticular RF-positive JIA also showed the highest aSvdH scores (median 222, range 0-313) (Table 2 and Fig. 2). Their scores were significantly higher than the scores from patients with polyarticular RF-negative JIA (median 22, $p=0.038$ ), extended oligoarticular JIA (median 3, $p=0.028$ ), enthesitis related arthritis (ERA) (median 0, $p=0.019$ ) and psoriatic arthritis (PsA) (median 1, $p=0.028$ ). Likewise, patients with polyarticular RF-positive JIA had significantly worse erosion and JSN scores (median 127 and 95, respectively) than those with polyarticular RF-negative JIA, extended oligoarticular JIA, ERA and PsA $(p \leq 0.05)$. The aSvdH scores were also significantly worse in anti-CCP-positive patients compared to anti-CCP-negative (mean 238 vs 2, $p=0.001)$.th $=\mathrm{tl} \mathrm{b}=$

We found that 2 patients (3\%) had only erosions, 2 patients (3\%) had only JSN, 29 patients (48\%) had both erosions and JSN (scores $>0$ ) and 27 patients (45\%) had no radiographic damage. The total erosion score correlated with the total JSN score $\left(r_{\mathrm{s}}=0.945, p<0.001\right)$ and the erosion scores in right and left hand correlated with the respective JSN scores $\left(r_{\mathrm{s}}=0.950\right.$ and $r_{\mathrm{s}}=0.897$, respectively, $p<0.001$ ).

When dividing radiographic damage into carpal area, MCP and PIP joints, we found that 31 patients (52\%) had damage in the carpal area, 26 (43\%) had in MCP joints and $24(40 \%)$ had damage in PIP joints $(p<0.001)$.

The mean CHR was $0.49 \pm 0.08$ (Table 3 ). The patients with polyarticular RF-positive JIA had significantly lower CHR than patients with polyarticular RF-negative JIA, persistent oligoarticular JIA, extended oligoarticular JIA, ERA and PsA ( $p \leq 0.005$ for all). Patients with systemic JIA had significantly lower CHR than patients with persistent oligoarticular JIA, ERA and PsA $(p<0.05)$. Eight patients had a mean score $\leq 0.38$. The mean CHR correlated significantly with aSvdH scores $\left(r_{\mathrm{s}}=-0,779\right.$ $p<0.001)$.

\section{The relation between radiographic scores and measures of disease activity and damage}

After 29 years, the total aSvdH, erosion and JSN scores correlated strongly with JADI scores and the number of joints with LROM ( $\mathrm{r}_{\mathrm{s}}=0.659$ to $0.721, p<0.001$, Table 4$)$. The CHR had also a high correlation with JADI scores and joints with LROM $\left(\mathrm{r}_{\mathrm{s}}=-0.660\right.$ to-0.645, $\left.p<0.001\right)$. The aSvdH score had weak correlations with JADAS-71 and CRP $\left(r_{\mathrm{s}}=0.266\right.$ to $\left.0.268, p<0.05\right)$. The erosion and JSN scores correlated with HAQ $\left(\mathrm{r}_{\mathrm{s}}=0.278\right.$ and $\mathrm{r}_{\mathrm{s}}=$ 0.259 , respectively, $p<0.05)$. All the radiographic scores of the hands/wrists had a weak to moderate correlation with vitamin A levels $\left(r_{\mathrm{s}}=-0.299\right.$ to $\left.0.430, p<0.05\right)$. Vitamin D levels did not correlate consistently with any of the scores, nor did the number of swollen, tender or active joins, pain, ESR, well-being or variables on smoking habits.

The aSvdH score for those in remission at 29 years was significantly lower than the score for those with active disease [median 0 (range $0-45$ ) vs median 11.5 (range $0-313), p=0.038]$. When analysing JSN scores separately, there was a significantly lower JSN score in 
Table 2 The aSvdH score in hands and wrists of patients with JIA at 29-year follow-up

\begin{tabular}{|c|c|c|c|c|c|c|c|c|}
\hline JIA categories & No. of patients & $\begin{array}{l}\text { No. of patients } \\
\text { with a score }>0\end{array}$ & $\begin{array}{l}\text { Total aSvdH } \\
\text { score }\end{array}$ & Erosion score & JSN score & $\begin{array}{l}\text { JADI } \\
\text { Articular damage }\end{array}$ & $\begin{array}{l}\text { JADI } \\
\text { Extra-articular } \\
\text { damage }\end{array}$ & $\begin{array}{l}\text { JADI } \\
\text { Total damage }\end{array}$ \\
\hline Total & $60(100)$ & $33(55)$ & $4(0-313)$ & $1(0-205)$ & $3(0-110)$ & $5(0-68)$ & $1(0-7)$ & $6(0-73)$ \\
\hline Systemic arthritis & $3(5)$ & $3(100)$ & $45(28-121)$ & $25(10-75)$ & $20(18-46)$ & $13(8-24)$ & $1(0-2)$ & $13(9-26)$ \\
\hline $\begin{array}{l}\text { Polyarticular } \\
\text { RF-negative }\end{array}$ & $11(18)$ & $8(61)$ & $22(0-131)$ & $5(0-76)$ & $8(0-69)$ & $8(0-36)$ & $1(0-2)$ & $6(0-38)$ \\
\hline $\begin{array}{l}\text { Polyarticular } \\
\text { RF-positive }\end{array}$ & $5(8)$ & $4(80)$ & $222(0-313)^{a}$ & $127(0-205)^{b}$ & $95(0-108)^{b}$ & $30(0-68)$ & $1(0-5)$ & $31(0-73)$ \\
\hline $\begin{array}{l}\text { Persistent } \\
\text { oligoarticular }\end{array}$ & $5(8)$ & $1(20)$ & $0(0-3)$ & $0(0-1)$ & $0(0-2)$ & 0 & $1(0-3)$ & $1(0-3)$ \\
\hline $\begin{array}{l}\text { Extended } \\
\text { oligoarticular }\end{array}$ & $10(17)$ & $5(50)$ & $3(0-95)$ & $0(0-56)$ & $3(0-39)$ & $7(0-24)$ & $1(0-3)$ & $8(0-24)$ \\
\hline $\begin{array}{l}\text { Enthesitis related } \\
\text { arthritis }\end{array}$ & $13(22)$ & $5(38)$ & $0(0-117)$ & $0(0-69)$ & $0(0-48)$ & $3(8-28)$ & $1(0-3)$ & $5(0-30)$ \\
\hline Psoriatic arthritis & $10(17)$ & $5(50)$ & $1(0-115)$ & $1(0-68)$ & $0(0-47)$ & $3(0-42)$ & $0(0-2)$ & $3(0-42)$ \\
\hline $\begin{array}{l}\text { Undifferentiated } \\
\text { arthritis }\end{array}$ & $3(5)$ & $2(67)$ & $31(0-308)$ & $25(0-128)$ & $6(0-110)$ & $7(2-43)$ & $4(0-7)$ & $11(2-50)$ \\
\hline
\end{tabular}

Numbers are median (range) or number (\%). ${ }^{a}$ Significantly different from scores in patients with polyarticular RF-negative JIA, extended oligoarticular JIA, enthesitis related arthritis and psoriatic arthritis (all $p<0.05$ ). ${ }^{\mathrm{b}}$ Significantly different from scores in patients with polyarticular RF-negative JIA, persistent oligoarticular JIA, extended oligoarticular JIA, enthesitis related arthritis and psoriatic arthritis (all $p<0.05$ ). aSvdH adapted Sharp van der Heijde, JSN joint space narrowing, JADI juvenile arthritis damage index

those in remission compared to those with active disease [median 0 (range 0-20) vs median 6 (range 0-110), $p=0.023]$. We found the same tendency for erosions, but the difference was not statistically significant $(p=0.054)$.

At 15 -year follow-up, $53(88 \%)$ of the 60 patients had used median 2.5 (range 1-7) different synthetic(s) DMARDs during their disease course (data not shown). At 29 years, 33 (69\%) out of total 48 patients with active disease, used sDMARDs, biological immunosuppressants and/or prednisolone. When comparing these patients with those not treated with sDMARDs, biological immunosuppressants and/or prednisolone $(n=15)$, those on treatment had significantly higher aSvdH scores [median 28 (range 0-313) vs median 0 (range $0-113$ ), $p=0.014$ ], but there were no significant differences in disease activity measures (ESR, CRP, number of active joints, joints with

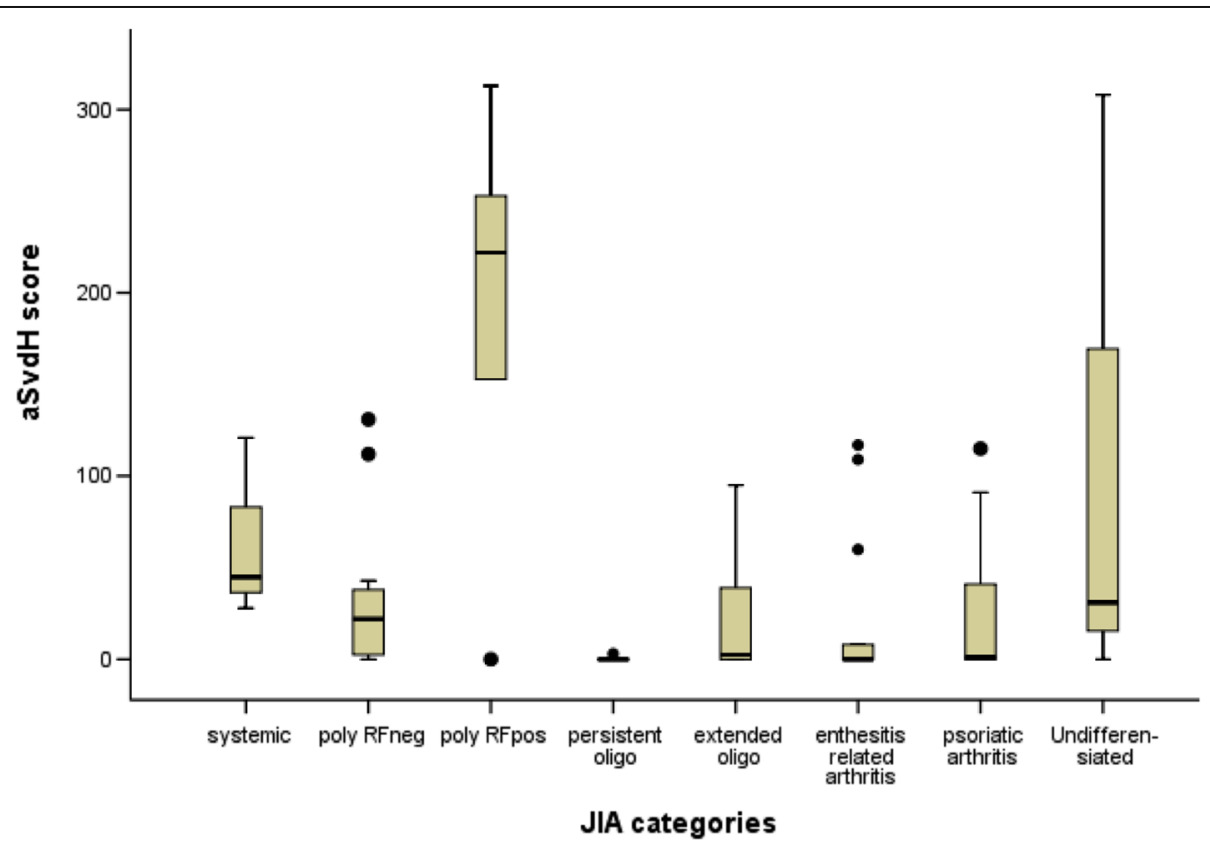

Fig. 2 The distribution of the aSvdH scores according to JIA category. aSvdH= adapted Sharp van der Heijde, RF = rheumatoid factor 
Table 3 The CHR of patients with JIA at 29-year follow-up

\begin{tabular}{lllll}
\hline JIA categories & No. of patients & CHR Right & CHR Left & Mean CHR \\
Total & $60(100)$ & $0.52(0.31-.64)$ & $0.52(0.31-0.63)$ & $0.49(0.08)$ \\
\hline Systemic arthritis & $3(5)$ & $0.32(0.31-0.48)$ & $0.34(0.31-0.48)$ & $0.37(0.09)^{\mathrm{a}}$ \\
Polyarticular RF-negative & $11(18)$ & $0.51(0.43-0.57)$ & $0.51(0.31-0.59)$ & $0.50(0.05)$ \\
Polyarticular RF-positive & $5(8)$ & $0.31(0.31-0.53)$ & $0.31(0.31-0.53)$ & $0.35(0.10)^{b}$ \\
Persistent oligoarticular & $5(8)$ & $0.56(0.52-0.58)$ & $0.56(0.52-0.56)$ & $0.55(0.02)$ \\
Extended oligoarticular & $10(17)$ & $0.50(0.31-0.55)$ & $0.51(0.47-0.56)$ & $0.50(0.04)$ \\
Enthesitis related arthritis & $13(22)$ & $0.55(0.42-0.64)$ & $0.54(0.31-0.62)$ & $0.54(0.06)$ \\
Psoriatic arthritis & $10(17)$ & $0.52(0.32-0.62)$ & $0.52(0.45-0.63)$ & $0.52(0.06)$ \\
Undifferentiated arthritis & $3(5)$ & $0.40(0.31-0.53)$ & $0.40(0.31-0.58)$ & $0.42(0.12)$ \\
\hline
\end{tabular}

Numbers are median (range), number (\%) or mean (SD). ${ }^{a}$ Significantly different from scores in patients with persistent oligoarticular arthritis, enthesitis related arthritis and psoriatic arthritis $(p<0.05)$. 'Significantly different from scores in patients with polyarticular RF-negative JIA, persistent oligoarticular JIA, extended oligoarticular JIA, enthesitis related arthritis and psoriatic arthritis $(p \leq 0.005)$. CHR carpal height ratio

LROM, patient's and physician's global assessment). There were no significant differences between aSvdH score or CHR in those employed versus those not employed (data not shown).

\section{Predictors of radiographic scores from clinical variables at baseline and 15 years}

All the core set variables from 15-year follow-up (except patient's global assessment of well-being), HLA-DR4, HLA-B27, onset and course type were associated with the aSvdH scores and CHR after 29 years (Table 5). In a multivariate linear regression analysis a higher number of joints with LROM and ESR from 15-year follow-up, predicted a higher aSvdH score at 29-year follow-up, $\mathrm{R}^{2}=65 \%$. The number of joints with LROM and HAQ at 15 years and HLA-B27 positivity were predictors of the mean $\mathrm{CHR}$ at 29 years, $\mathrm{R}^{2}=51 \%$.

\section{Discussion}

In the present study of 60 patients with long-term active JIA and clinical arthritis in the wrist during their disease course, more than half of these patients had radiographic damage measured by the aSvdH score in the hands/ wrists after 29 years. The damage was modest in most patients, but $25 \%$ had severe damage. Patients with polyarticular RF-positive or anti-CCP-positive JIA had the worst damage. The majority of patients with radiographic damage had both erosions and JSN. Most damage occurred in the carpus area compared to the rest of the hand. Both the radiographic scores correlated strongly with other measures of disease damage at 29year follow-up. The number of joints with LROM at 15 years was the most important predictor of the radiographic scores after 29 years of disease duration.

We found that $55 \%$ of the patients with long-term active disease and previous or present arthritis in the wrist had developed radiographic damage in the hands/wrists. There are few studies on radiographic long-term outcome in JIA patients, and reports of radiographic outcome especially in hands/wrists are even more scarce. A study from the sixties reported presence of erosions in hands/wrists of $68 \%$ of patients with juvenile rheumatoid arthritis (JRA) after 18 years [26]. The majority of their patients had polyarticular disease and $28 \%$ of them were RF-positive. Damage in this study refers to erosions only which is not comparable to the aSvdH score. Another study reported radiographic damage in hands/wrists in $79 \%$ of patients with polyarticular JIA (54\% RF-positive)

Table 4 Correlations between radiographic scores and measures of disease damage and disease activity at 29-year follow-up

\begin{tabular}{lllll}
\hline & aSvdH scores & Erosion scores & JSN scores & Mean CHR \\
Variables & $4.0(0-313)$ & $1.0(0-205)$ & $3.0(0-110)$ & $0.52(0.31-0.63)$ \\
\hline JADI total & $0.706^{\mathrm{a}}$ & $0.721^{\mathrm{a}}$ & $0.714^{\mathrm{a}}$ & $-0.660^{\mathrm{a}}$ \\
Joints with LROM & $0.669^{\mathrm{a}}$ & $0.659^{\mathrm{a}}$ & $0.665^{\mathrm{a}}$ & $-0.645^{\mathrm{a}}$ \\
JADAS & $0.268^{\mathrm{b}}$ & $0.255^{\mathrm{b}}$ & $0.266^{\mathrm{b}}$ & $\mathrm{NS}$ \\
HAQ & $\mathrm{NS}$ & $0.278^{\mathrm{b}}$ & $0.259^{\mathrm{b}}$ & $\mathrm{NS}$ \\
CRP & $0.266^{\mathrm{b}}$ & $\mathrm{NS}$ & $0.273^{\mathrm{b}}$ & $\mathrm{NS}$ \\
Vitamin A & $-0.272^{\mathrm{b}}$ & $-0.275^{\mathrm{b}}$ & $-0.299^{\mathrm{b}}$ & $0.430^{\mathrm{a}}$ \\
\hline
\end{tabular}

Numbers are median scores (range) and Spearman's correlation coefficients. The radiographic scores did not correlate with the number of swollen, tender and active joints, pain, well-being, ESR or vitamin D. ${ }^{a} p \leq 0.001,{ }^{b} p<0.05$. aSvdH adapted Sharp van der Heijde, JSN joint space narrowing, CHR carpal height ratio, $J A D I$ juvenile arthritis damage index, LROM limited range of motion, JADAS juvenile arthritis disease activity score, NS not significant 


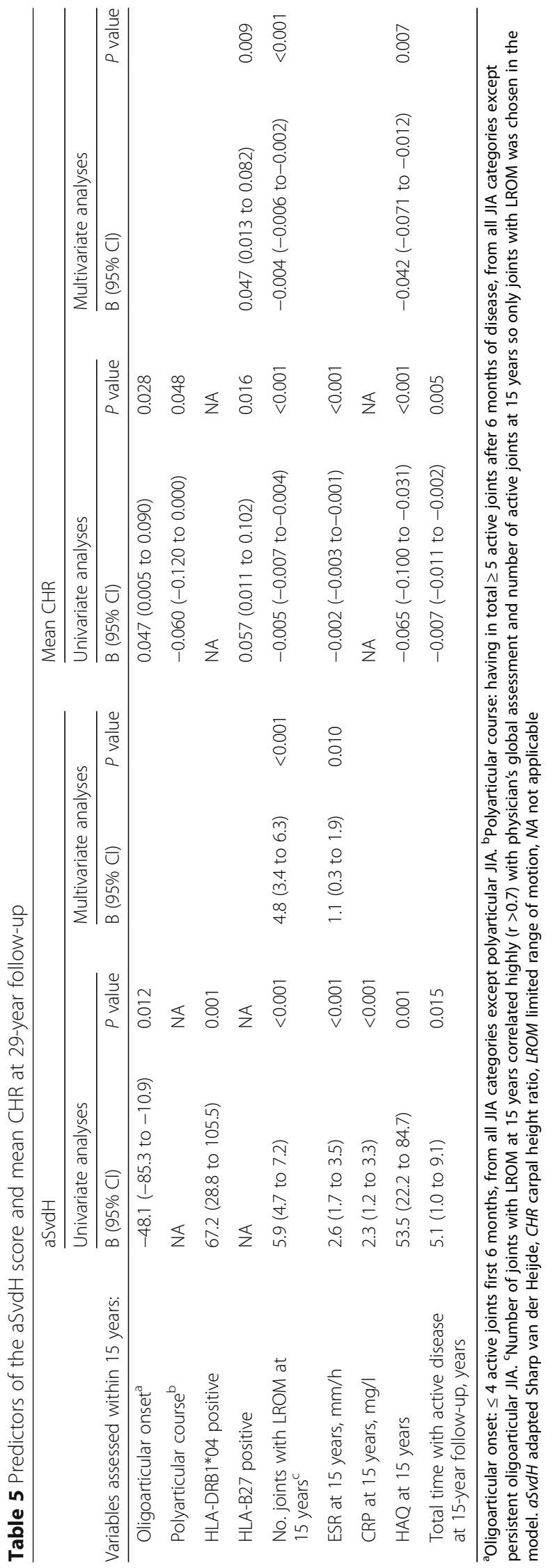


after 13.3 years compared to $86 \%$ in patients with RA (79\% RF-positive, not significant) after 11 years [27]. Other studies have reported worse radiographic outcome in patients with RA compared to patients with JIA [28].

Patients with polyarticular RF-positive JIA showed the most serious damage in hands/wrists and most of them had been through surgical operations in the wrist. It is well known that patients in this JIA category have the most aggressive disease and thus the most damage [19, 29-32]. Studies have shown that after 10-15 years of disease duration the most severely affected joints in RFpositive polyarticular juvenile arthritis were hands/ wrists, and erosions were most commonly seen in these joints $[1,8,27,33]$. We found that $\mathrm{aSvdH}$ scores were significantly worse in anti-CCP-positive patients compared to anti-CCP-negative patients, which is in harmony with other studies $[34,35]$.

In the present study most patients (29/33) with radiographic damage had both erosions and JSN after 29 years and very few had only erosions or JSN. Erosion and JSN scores correlated highly. There are few studies that have assessed this issue up to now in JIA [36]. One study found that erosions and JSN occurred at almost equal frequencies in patients with polyarticular JRA examined after 8 years of disease [32].

Patients had more often radiographic damage in the carpal area than in MCPs and PIPs in our study. A recent study supports this as they reported more damage in carpus and less in MCPs after up to 10 years of disease duration in patients with JIA and polyarticular course [36]. On the other hand, another study found damage in the MCP joints of all patients with polyarticular JIA after 13 years of disease duration and in $97 \%$ of the carpal area [27].

We chose to include the $\mathrm{CHR}$ to compare radiographic damage in the carpal area as measured by aSvdH and CHR. Both methods defined patients with polyarticular RF-positive JIA as having the worst damage and patients with systemic JIA as having the second worst damage. A study which applied the Poznanski method also found significant damage in the wrist of these two JIA categories [37]. The aSvdH scores correlated strongly with the CHR, in the same way as other studies have shown moderate to high correlations between the Sharp/ aSvdH and the Poznanski score $[6,10]$. Thus the aSvdH score performs well in scoring wrist damage in JIA.

We observed high correlations between the radiographic scores aSvdH or CHR and scores for disease damage (JADI and joints with LROM), and lower correlations for the disease activity score (JADAS-71). This is in harmony with other studies on JIA which have reported associations between radiographic measures and JADI and with the number of joints with LROM [6, 10, 11, 21, 32, 38]. Also in adult RA radiographic scores are reported to be associated with other measures of disease damage and disability $[39,40]$.

Eighty percent of our patients had active disease, but only $69 \%$ of them used sDMARDs, biological immunosuppressants and/or prednisolone. This may indicate that some of them are not optimally treated, but on the other hand they may have a mild disease course as the aSvdH scores of those not on advanced treatment were in the lower range.

There were persistent correlations between vitamin A levels and all the radiographic scores. Fifteen percent of the patients had vitamin A levels $\leq 1.2 \mu \mathrm{mol} / \mathrm{L}$ and this subgroup had the highest radiographic scores. Retinoic acid is known to be involved in proliferation and differentiation of osteoblasts and low levels of retinoic acid can have a harmful effect on bone metabolism $[41,42]$. However, this correlation needs to be confirmed. There is a lot of research in the field of retinoic acid and its importance in regulation of $\mathrm{T}$ cells and other immune cells $[43,44]$. Alternatively, our finding of an association with low vitamin A status may be connected to deficiency in immune cells and thus the inflammatory process and not directly on bone metabolism.

We found that the number of joints with LROM and ESR from 15-year follow-up were predictors of aSvdH after 29 years. This is supported by previous studies, which have found ESR as an important predictor for joint destruction $[45,46]$. We have in an earlier 3-year follow-up study, revealed the number of joints with LROM as a predictor of radiographic changes [7]. Others have reported that continuing disease activity is a predictor of joint damage [47]. HLA-B27 positivity protected against radiographic damage measured as CHR. This seems to be explained by the fact that 11 out of 18 HLAB27 positive patients had ERA and they had less damage in the wrists as shown in Tables 2 and 3. Alternatively HLA-B27 represents a marker for ERA. When ERA patients were excluded from the analysis, HLA-B27 was no longer a predictor of CHR. On the other hand, a previous study found HLA-B27 positivity associated with worse radiographic outcome in JIA [9].

The strength of the study is the long-term follow-up over 29 years of this cohort that has been described in several publications $[17-19,46,48]$. There are however some limitations of the study. We have examined only patients with long-term active disease who also had current or previous clinical arthritis in the wrist joints, thus a selection of those with severe disease. Optimally we could have taken radiographs of all the patients, including those who went into remission before 15 years. We would have registered the progression of bone damage compared to previous radiographs, but these were unfortunately not available. There were few patients with persistent oligoarticular JIA, therefore their results must 
be interpreted with caution. The radiographic method we used, the aSvdH score, is evaluated for children with JIA, but we have used it in adults with JIA. However, the Sharp van der Heijde method is widely used in adults with RA and the main difference in this adapted version is the adding of locations in the carpal area in which patients with JIA have more changes than adults with RA [10]. Another limitation is that the JADAS is not validated for adults with JIA. On the other hand, we used the JADAS in a recent study and found that the JADAS correlated strongly with categories of disease activity in adult patients, but JADAS may capture some other aspects of the burden of disease like damage and overall well-being in adults [19].

We did not perform intra- and interrater reliability testing among raters of the radiographs. Our two raters agreed by consensus. The Sharp/Sharp van der Heijde method has previously been evaluated as having good reliability and reproducibility $[6,49]$, and a good interand intraobserver agreement has been demonstrated for the aSvdH in JIA [10].

\section{Conclusions}

In the present cohort of patients with prolonged active JIA and wrist involvement during 29 years of disease duration, we found that about half of the patients had radiographic damage in hands/wrists. Changes were modest for most patients, but one out of four had severe damage. Patients with JIA and arthritis in hands/wrist should be examined regularly with radiographs and especially patients who have joints with LROM. Our patients were diagnosed early in the methotrexate era. Patients nowadays receive more aggressive therapy and will most likely have better radiographic outcome in long-term follow-up than patients in the present study. Similar longterm radiographic outcome studies are needed to continually survey the effectiveness of the present medical regimes, and hopefully document improved outcomes compared to the present study in the future.

\footnotetext{
Abbreviations

ANA: Antinuclear antibody; anti-CCP: Anti-cyclic citrullinated peptides; aSvdH score: The adapted version of the Sharp van der Heijde score; CHR: Carpal height ratio; CRP: C-reactive protein; ERA: Enthesitis related arthritis; ESR: Erythrocyte sedimentation rate; HAQ: Health assessment questionnaire; HLA: Human leukocyte antigen; ILAR: International League of Associations for Rheumatology; JADAS: Juvenile arthritis disease activity score; JADI: Juvenile arthritis damage index; JIA: Juvenile idiopathic arthritis; JSN: Joint space narrowing; LROM: Limited range of motion; MCP: Metacarpophalangeal; PIP: Proximal interphalangeal; PSA: Psoriatic arthritis; RA: Rheumatoid arthritis; RF: Rheumatoid factor; sDMARDs: Synthetic disease-modifying anti-rheumatic drugs; SF-36: Medical outcome study 36-item short form health survey; VAS: Visual analogue scale
}

\section{Acknowledgements}

The authors thank Torhild Garen for assistance in data management, the Hormone Laboratory, Aker, for the analyses of vitamin D and the Department of Medical Biochemistry at Oslo University Hospital for the analyses of vitamin A.
Funding

The study was funded by the Norwegian Women's Public Health Association (H3/2012).

\section{Availability of data and materials}

The datasets generated and analyzed during the current study are not publicly available due to strict ethical regulation of health related data in Norway. The consent to participate does not include permission to make the data available to a third party.

\section{Authors' contributions}

$\mathrm{BF}, \mathrm{EK}, \mathrm{HAA}$ and $\mathrm{AMS}$ contributed to the design and conception of the study. $B F, V L, H A A$, and AMS administered and examined the patients clinically. EK and LT scored the radiographs. AMS analyzed the data and drafted the manuscript. All authors revised the manuscript critically. All authors read and approved the final manuscript.

\section{Competing interests}

The authors declare that they have no competing interests.

\section{Consent for publication}

Not applicable.

\section{Ethics approval and consent to participate}

The study was approved by the Regional Committee for Medical and Health Research Ethics, 2011/982/REK sør-øst. Written informed consent was obtained from all the participants.

\section{Publisher's Note}

Springer Nature remains neutral with regard to jurisdictional claims in published maps and institutional affiliations.

\section{Author details}

${ }^{1}$ Department of Rheumatology, Oslo University Hospital, Rikshospitalet, Post-box 4950 Nydalen, 0424 Oslo, Norway. ${ }^{2}$ Department of Radiology and Nuclear Medicine, Oslo University Hospital, Rikshospitalet, Oslo, Norway. ${ }^{3}$ Institute of Clinical Medicine, Faculty of Medicine, University of Oslo, Oslo, Norway. ${ }^{4}$ Present address: Department of Radiology, Linköping University, Linköping, Sweden. ${ }^{5}$ Present address: Department of Internal Medicine,

Diakonhjemmet Hospital, Oslo, Norway.

Received: 18 November 2016 Accepted: 23 March 2017

Published online: 11 April 2017

\section{References}

1. Ravelli A. The time has come to include assessment of radiographic progression in juvenile idiopathic arthritis clinical trials. J Rheumatol. 2008; 35:553-7.

2. van Rossum MA, Boers M, Zwinderman $A H$, van Soesbergen RM, Wieringa $H$, Fiselier TJ, et al. Development of a standardized method of assessment of radiographs and radiographic change in juvenile idiopathic arthritis: introduction of the Dijkstra composite score. Arthritis Rheum. 2005;52:2865-72.

3. Dale K, Paus AC, Laires K. A radiographic classification system in juvenile rheumatoid arthritis applied to the knee. Eur Radiol. 1994:4:27-32.

4. Poznanski AK, Hernandez RJ, Guire KE, Bereza UL, Garn SM. Carpal length in children-a useful measurement in the diagnosis of rheumatoid arthritis and some concenital malformation syndromes. Radiology. 1978;129:661-8.

5. Magni-Manzoni S, Rossi F, Pistorio A, Temporini F, Viola S, Beluffi G, et al. Prognostic factors for radiographic progression, radiographic damage, and disability in juvenile idiopathic arthritis. Arthritis Rheum. 2003;48:3509-17.

6. Rossi F, Di Dia F, Galipo O, Pistorio A, Valle M, Magni-Manzoni S, et al. Use of the Sharp and Larsen scoring methods in the assessment of radiographic progression in juvenile idiopathic arthritis. Arthritis Rheum. 2006;55:717-23.

7. Selvaag AM, Flato B, Dale K, Lien G, Vinje O, Smerdel-Ramoya A, et al. Radiographic and clinical outcome in early juvenile rheumatoid arthritis and juvenile spondyloarthropathy: a 3-year prospective study. J Rheumatol. 2006:33:1382-91.

8. Mason T, Reed AM, Nelson AM, Thomas KB, Patton A, Hoffman AD, et al. Frequency of abnormal hand and wrist radiographs at time of diagnosis of polyarticular juvenile rheumatoid arthritis. J Rheumatol. 2002;29:2214-8. 
9. van Rossum MA, Zwinderman AH, Boers M, Dijkmans BA, van Soesbergen RM, Fiselier TJ, et al. Radiologic features in juvenile idiopathic arthritis: a first step in the development of a standardized assessment method. Arthritis Rheum. 2003:48:507-15.

10. Ravelli A, loseliani M, Norambuena X, Sato J, Pistorio A, Rossi F, et al. Adapted versions of the Sharp/van der Heijde score are reliable and valid for assessment of radiographic progression in juvenile idiopathic arthritis. Arthritis Rheum. 2007;56:3087-95.

11. Palmisani E, Solari N, Magni-Manzoni S, Pistorio A, Labo E, Panigada S, et al. Correlation between juvenile idiopathic arthritis activity and damage measures in early, advanced, and longstanding disease. Arthritis Rheum. 2006;55:843-9.

12. Aoki C, Inaba Y, Choe H, Kaneko U, Hara R, Miyamae T, et al. Discrepancy between clinical and radiological responses to tocilizumab treatment in patients with systemic-onset juvenile idiopathic arthritis. J Rheumatol. 2014; 41:1171-7.

13. Youm Y, McMurthy R, Flatt AE, Gillespie TE. Kinematics of the wrist. I. An experimental study of radial-ulnar deviation and flexion-extension. J Bone Joint Surg Am. 1978;60:423-31.

14. Feipel V, Rinnen D, Rooze M. Postero-anterior radiography of the wrist. Normal database of carpal measurements. Surg Radiol Anat. 1998;20:221-6.

15. Jeffery LE, Raza K, Hewison M. Vitamin D in rheumatoid arthritis-towards clinical application. Nat Rev Rheumatol. 2016;12:201-10.

16. Dadon Bar-El S, Reifen R. Vitamin A and the Epigenome. Crit Rev Food Sci Nutr. 2015. doi:10.1080/10408398.2015.1060940.

17. Flato B, Lien G, Smerdel A, Vinje O, Dale K, Johnston V, et al. Prognostic factors in juvenile rheumatoid arthritis: a case-control study revealing early predictors and outcome after 14.9 years. J Rheumatol. 2003;30:386-93.

18. Flato B, Hoffmann-Vold AM, Reiff A, Forre O, Lien G, Vinje O. Long-term outcome and prognostic factors in enthesitis-related arthritis: a case-control study. Arthritis Rheum. 2006;54:3573-82.

19. Selvaag $A M$, Aulie HA, Lilleby $V$, Flato B. Disease progression into adulthood and predictors of long-term active disease in juvenile idiopathic arthritis. Ann Rheum Dis. 2016;75:190-5.

20. Petty RE, Southwood TR, Manners P, Baum J, Glass DN, Goldenberg J, et al. International League of Associations for Rheumatology classification of juvenile idiopathic arthritis: second revision, Edmonton, 2001. J Rheumatol. 2004:31:390-2.

21. Viola S, Felici E, Magni-Manzoni S, Pistorio A, Buoncompagni A, Ruperto N, et al. Development and validation of a clinical index for assessment of long-term damage in juvenile idiopathic arthritis. Arthritis Rheum. 2005;52:2092-102.

22. Fries JF, Spitz P, Kraines RG, Holman HR. Measurement of patient outcome in arthritis. Arthritis Rheum. 1980;23:137-45.

23. Consolaro A, Ruperto N, Bazso A, Pistorio A, Magni-Manzoni S, Filocamo G, et al. Development and validation of a composite disease activity score for juvenile idiopathic arthritis. Arthritis Rheum. 2009;61:658-66.

24. Loge JH, Kaasa S, Hjermstad MJ, Kvien TK. Translation and performance of the Norwegian SF-36 Health Survey in patients with rheumatoid arthritis. I. Data quality, scaling assumptions, reliability, and construct validity. J Clin Epidemiol. 1998:51:1069-76.

25. Giannini EH, Ruperto N, Ravelli A, Lovell DJ, Felson DT, Martini A. Preliminary definition of improvement in juvenile arthritis. Arthritis Rheum. 1997;40: $1202-9$.

26. Jeremy R, Schaller J, Arkless R, Wedgwood RJ, Healey LA. Juvenile rheumatoid arthritis persisting inot adulthood. Am J Med. 1968:45:419-34

27. Elhai M, Bazeli R, Freire V, Feydy A, Drape JL, Quartier P, et al. Radiological peripheral involvement in a cohort of patients with polyarticular juvenile idiopathic arthritis at adulthood. J Rheumatol. 2013;40:520-7.

28. Koivuniemi R, Leirisalo-Repo M. Juvenile chronic arthritis in adult life: a study of long-term outcome in patients with juvenile chronic arthritis or adult rheumatoid arthritis. Clin Rheumatol. 1999;18:220-6.

29. Wallace CA, Huang B, Bandeira M, Ravelli A, Giannini EH. Patterns of clinical remission in select categories of juvenile idiopathic arthritis. Arthritis Rheum. 2005:52:3554-62.

30. Ringold S, Seidel KD, Koepsell TD, Wallace CA. Inactive disease in polyarticular juvenile idiopathic arthritis: current patterns and associations. Rheumatology (Oxford). 2009;48:972-7.

31. Guzman J, Oen K, Tucker LB, Huber AM, Shiff N, Boire G, et al. The outcomes of juvenile idiopathic arthritis in children managed with contemporary treatments: results from the ReACCh-Out cohort. Ann Rheum Dis. 2015;74:1854-60.
32. Oen K, Reed M, Malleson PN, Cabral DA, Petty RE, Rosenberg AM, et al. Radiologic outcome and its relationship to functional disability in juvenile rheumatoid arthritis. J Rheumatol. 2003;30:832-40.

33. Williams RA, Ansell BM. Radiological findings in seropositive juvenile chronic arthritis (juvenile rheumatoid arthritis) with particular reference to progression. Ann Rheum Dis. 1985:44:685-93.

34. Lipinska J, Brozik H, Stanczyk J, Smolewska E. Anticitrullinated protein antibodies and radiological progression in juvenile idiopathic arthritis. J Rheumatol. 2012;39:1078-87.

35. Omar A, Abo-Elyoun I, Hussein H, Nabih M, Atwa H, Gad S, et al. Anti-cyclic citrullinated peptide (anti-CCP) antibody in juvenile idiopathic arthritis (JIA): correlations with disease activity and severity of joint damage (a multicenter trial). Joint Bone Spine. 2013;80:38-43.

36. Giancane G, Pederzoli S, Norambuena X, loseliani M, Sato J, Gallo MC, et al. Frequency of radiographic damage and progression in individual joints in children with juvenile idiopathic arthritis. Arthritis Care Res (Hoboken). 2014; 66:27-33.

37. Ozawa R, Inaba Y, Mori M, Hara R, Kikuchi M, Higuchi R, et al. Definitive differences in laboratory and radiological characteristics between two subtypes of juvenile idiopathic arthritis: systemic arthritis and polyarthritis. Mod Rheumatol. 2012;22:558-64.

38. Susic GZ, Stojanovic RM, Pejnovic NN, Damjanov NS, Soldatovic II, Jablanovic DB, et al. Analysis of disease activity, functional disability and articular damage in patients with juvenile idiopathic arthritis: a prospective outcome study. Clin Exp Rheumatol. 2011;29:337-44.

39. Bejia I, Braham R, Said M, Salem KB, Touzi M, Bergaoui N. Reliability of the rheumatoid arthritis articular damage score in Tunisian patients. Clin Rheumatol. 2005;24:593-4.

40. Odegard S, Landewe R, van der Heijde D, Kvien TK, Mowinckel P, Uhlig T. Association of early radiographic damage with impaired physical function in rheumatoid arthritis: a ten-year, longitudinal observational study in 238 patients. Arthritis Rheum. 2006;54:68-75.

41. Bosetti M, Sabbatini M, Calarco A, Borrone A, Peluso G, Cannas M. Effect of retinoic acid and vitamin D3 on osteoblast differentiation and activity in aging. J Bone Miner Metab. 2016;34:65-78.

42. Tanaka K, Tanaka S, Sakai A, Ninomiya T, Arai Y, Nakamura T. Deficiency of vitamin A delays bone healing process in association with reduced BMP2 expression after drill-hole injury in mice. Bone. 2010;47:1006-12.

43. Brown CC, Esterhazy D, Sarde A, London M, Pullabhatla V, Osma-Garcia I, et al. Retinoic acid is essential for Th1 cell lineage stability and prevents transition to a Th17 cell program. Immunity. 2015;42:499-511.

44. Chatterjee A, Gogolak P, Blottiere HM, Rajnavolgyi E. The impact of ATRA on shaping human myeloid cell responses to epithelial cell-derived stimuli and on T-lymphocyte polarization. Mediators Inflamm. 2015. doi:10.1155/2015/579830.

45. Guillaume S, Prieur AM, Coste J, Job-Deslandre C. Long-term outcome and prognosis in oligoarticular-onset juvenile idiopathic arthritis. Arthritis Rheum. 2000;43:1858-65.

46. Flato B, Smerdel A, Johnston V, Lien G, Dale K, Vinje O, et al. The influence of patient characteristics, disease variables, and HLA alleles on the development of radiographically evident sacroiliitis in juvenile idiopathic arthritis. Arthritis Rheum. 2002;46:986-94.

47. Gilliam BE, Chauhan AK, Low JM, Moore TL. Measurement of biomarkers in juvenile idiopathic arthritis patients and their significant association with disease severity: a comparative study. Clin Exp Rheumatol. 2008;26:492-7.

48. Aulie HA, Selvaag AM, Gunther A, Lilleby V, Molberg O, Hartmann A, et al. Arterial haemodynamics and coronary artery calcification in adult patients with juvenile idiopathic arthritis. Ann Rheum Dis. 2015;74:1515-21.

49. Boini S, Guillemin F. Radiographic scoring methods as outcome measures in rheumatoid arthritis: properties and advantages. Ann Rheum Dis. 2001;60: 817-27. 